Wieteska Małgorzata, Kaczyńska Agnieszka, Maj Dominik, Stanicki Pawel, Chałupnik Aleksandra, Wójcik Piotr. Metformin as a medicine decreasing cardiovascular risk in patients suffering from type 2 diabetes mellitus. Journal of Education, Health and Sport. 2020;10(8):105-111. eISSN 2391-8306. DOI http://dx.doi.org/10.12775/JEHS.2020.10.08.012

https://apcz.umk.pl/czasopisma/index.php/JEHS/article/view/JEHS.2020.10.08.012

https://zenodo.org/record/3971259

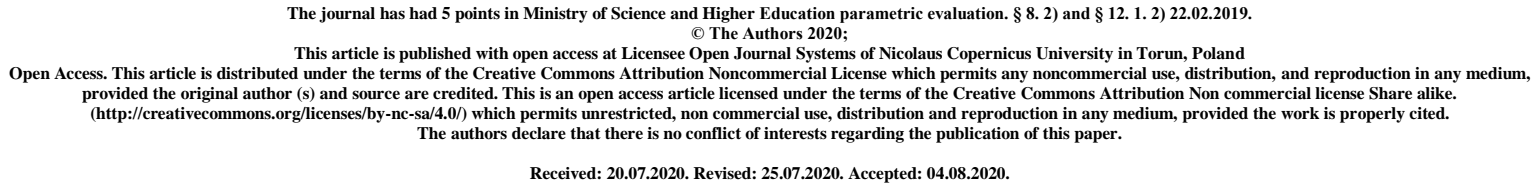

\title{
Metformin as a medicine decreasing cardiovascular risk in patients suffering from type 2 diabetes mellitus
}

\author{
Małgorzata Wieteska ${ }^{1}$, Agnieszka Kaczyńska ${ }^{1}$, Dominik Maj ${ }^{2}$, Pawel Stanicki ${ }^{1}$, \\ Aleksandra Chałupnik ${ }^{1}$, Piotr Wójcik ${ }^{1}$
}

(1) Student Research Circle at the Department of Epidemiology and Clinical Research Methodology, Medical University of Lublin, Poland

(2) Student Research Circle at the Department of Internal Medicine and Cardiology, Medical University of Warsaw, Poland

Corresponding author: Małgorzata Wieteska; malgosia.wieteska@onet.eu

ORCID ID and e-mail:

Małgorzata Wieteska: https://orcid.org/0000-0002-1566-7559; malgosia.wieteska@ onet.eu Agnieszka Kaczyńska: https://orcid.org/0000-0002-8943-9401; agaagnieszkakk@gmail.com Dominik Maj: https://orcid.org/0000-0001-5100-4130; domimaj9@gmail.com Paweł Stanicki: https://orcid.org/0000-0002-4942-9030; stanicki99@ gmail.com Aleksandra Chałupnik: https://orcid.org/0000-0003-4249-470X; olachalupnik@op.pl Piotr Wójcik: https://orcid.org/0000-0002-9078-3701; piotrek1444@gmail.com 


\begin{abstract}
Almost half of deaths in Poland (approximately 49\%) are caused by cardiovascular system diseases. It means that in 2018 it contributed to the death of over two hundred thousand people in our country. Myocardial infarction, stroke and heart failure are the most common of cardiovascular diseases (CVD). The main risk factor for progression of cardiovascular diseases is type 2 diabetes mellitus (T2D). It is estimated that almost 3 million people suffer from T2D in Poland. Metformin is a promising medicine that reduces cardiovascular risk in patients with T2D. It is currently used as the first line drug in patients suffering from T2D. The aim of the presented article is to review current research on the effectiveness of the metformin as a medicine that reduces cardiovascular risk in patients with T2D. Metformin is the oldest and most commonly used medicine that reduces the concentration of glucose in the peripheral blood in patients with T2D. Due to its very good safety profile, low cost and high effectiveness, metformin is used in the first-line treatment for T2D. Recent studies indicate its protective effect by vasodilation, reducing the size of myocardial infarction, inhibiting apoptosis of cardiomyocytes or reducing oxidative stress. Currently, metformin can be seen to have a great potential in the treatment of cardiovascular complications occurring in the course of T2D. Despite the fact that this medicine has been on the market for many years, the studies on its cardio protective effects have been carried out for a relatively short period of time. It is possible that the detailed understanding of all mechanisms of action of metformin will lead to future advances in the treatment of not only T2D, but also cardiovascular and cancer diseases.
\end{abstract}

Key words: Metformin; type 2 diabetes mellitus; cardiovascular diseases; myocardial infarction; atherosclerosis.

\title{
1. Introduction and purpose
}

Cardiovascular diseases are leading cause of death in Poland. According to the report of the Polish Society of Cardiology, heart failure is associated with huge social costs due to its negative impact on health and quality of life, inability to work, being heavy load on the nearest surrounding and poor long-term prognosis [1]. According to the latest researches, $32.2 \%$ of patients with T2D also suffer from cardiovascular diseases, the risk of which soars up due to patient' $s$ age [2]. Diabetes mellitus, defined as a group of metabolic diseases characterized by hyperglycemia, is a major risk factor for the development of cardiovascular diseases. Despite the progress on the field of diagnostic and therapeutic technologies, cardiovascular diseases still remain the most common cause of death among patients with T2D. Lifestyle modification in patients with T2D, as well as appropriate pharmacological therapy are the basis for the prevention of the development of cardiovascular diseases. Clinical care for those patients focuses on increasing their physical activity, as well as introducing a nutritional pattern based on a diet rich in products with a low glycemic index. The aim of taken actions is to normalise the body weight, which is measured by BMI (body mass index). Pharmacological therapy focuses on reducing the level of glucose in the patient's peripheral blood. The first line drug with a hypoglycaemic effect is metformin. It is an orally administered drug that is widely used, safe, and with few side effects. According to the latest researches, metformin may reduce cardiovascular risk in patients with $\mathrm{T} 2 \mathrm{D}$, and therefore in the future it may lead to the reduction of mortality due to cardiovascular diseases among 
patients with T2D [3]. Such a high rate of incidence of cardiovascular diseases in patients with T2D prompts to pay more attention to the future therapeutic possibilities that could be potential opportunities for patients. That kind of drug of hope for the future is metformin, which has a protective effect on the cardiovascular system. The aim of the presented article is to review current research on the effectiveness of the metformin as a medicine that reduces cardiovascular risk in patients with T2D.This article reviews the data available in the literature on cardiovascular diseases, in particular in patients with $\mathrm{T} 2 \mathrm{D}$, as well as the latest discoveries regarding metformin as a drug that reduces cardiovascular risk in patients with T2D. The information used in the presented analysis were obtained from searching academic research databases: Google Scholar and PubMed. The strategy of searching is based on the following keywords: metformin, type 2 diabetes mellitus, cardiovascular diseases, myocardial infarction and atherosclerosis. The available articles have been selected in terms of their content value and thematic connection with this article.

\section{Description of the state of knowledge}

\subsection{Cardiovascular diseases and type 2 diabetes mellitus}

Type 2 diabetes mellitus (T2D) is a major risk factor for the development of cardiovascular diseases, which remain the most common cause of death among patients with T2D [4]. According to the statistics, almost a third of patients suffering from T2D also struggle with cardiovascular diseases. T2D is a significant risk factor for stroke, angina pectoris, congestive heart failure, myocardial infarction, and atherosclerosis [5]. It is worth of attention that mortality caused by cardiovascular diseases is decreasing in highly developed countries, probably as a result of numerous highly effective preventive and therapeutic programs [2]. Other reasons include better hospital care, effective diagnostics and modification of behavioral risk factors, such as diet, tobacco smoking and alcohol consumption. This phenomenon can be a consequence of constantly increasing social consciousness [6]. The mechanisms leading to the development of atherosclerosis and congestive heart failure are exacerbated in patients with T2D. Some of those patients suffer from cardiovascular diseases with typical symptoms such as angina pectoris, overt heart failure syndrome, symptomatic vascular occlusive disease and symptomatic cardiac arrhythmia. However, there is a group of patients with T2D, accompanied by unrecognised diabetic cardiac impairment with atypical symptoms such as asymptomatic myocardial ischemia, asymptomatic heart failure or cardiac arrhythmia. UDCI (unrecognised diabetic cardiac impairment) is proposed as a standardised terminology that accurately accounts for this asymptomatic phenomenon. UDCI affects almost one third of patients with T2D. Diagnostic difficulties related to UDCI may lead to the development of negative consequences of undetected cardiovascular diseases. Increased awareness of the UDCI, both among healthcare professionals and patients, would allow the development of effective tools to screen patients with T2D for UDCI. Examples of these potential tools are: questionnaires to obtain detailed patient histories, digital data gathering for continuous patient monitoring, cardiac stress test and biomarkers such as BNP (brain natriuretic peptide) [7]. Researches have confirmed the existence of a common genetic basis underlying T2D and cardiovascular diseases. Both disease entity also have common development risk factors [8]. The risk of cardiovascular diseases development in patients with T2D is associated with hyperglycemia and other glycemic disorders. The presence of chronic 
inflammation, dyslipidemia, hypertension and other coexisting diseases are risk factors for the development of macrovascular diseases, in particular atherosclerosis in patients with T2D. The risk depends on the duration of diabetes and the age of the patient. The assessment of microangiopathic complications in patients with $\mathrm{T} 2 \mathrm{D}$ is correlated with carotid plaque score. Treatment of T2D should focus on normalizing the patients' blood glucose levels and minimizing the risk of cardiovascular events. These activities require the cooperation of doctors of many specialties: diabetologists, cardiologists, nephrologists and other healthcare professionals [9].

\subsection{Metformin}

The history of metformin as a pharmacological substance began in 1918, when it was discovered that metformin has the properties of lowering the concentration of glucose in the peripheral blood. Currently, it is the most widely used, orally administered, first-line hypoglycemic drug in patients with T2D [10]. The reasons for the superiority of metformin over other drugs that normalize blood glucose levels are its high effectiveness, low cost, good tolerance, a small number of side effects and overall good safety profile [11]. Commonly reported side effects of metformin are gastrointestinal, but these symptoms are generally transient and resolve spontaneously. Other symptoms are rather few. Serious side effects are seldom and limited to the lactic acidosis in patients with renal or hepatic insufficiency [12]. Metformin is contraindicated in patients with renal failure, heart failure, respiratory failure, severe liver disorders, tissue hypoxia, dehydration, acute and chronic alcohol abuse, diabetic ketoacidosis, diabetic precoma or in patients before i.v. iodinated contrast medium administration [13]. According to the guidelines of the American Diabetes Association and European Association for the Study of Diabetes the use of metformin is contraindicated if GFR (glomerular filtration rate) is $<30 \mathrm{~mL} / \mathrm{min} / 1.73 \mathrm{~m}^{2}$. The dose of metformin should be adapted and optionally reduced depending on the renal function [14]. However, studies suggest that metformin can still be used with caution in patients with mild to moderate chronic kidney disease in conditions of monitoring the glomerular filtration rate (GFR) [15]. Metformin exerts its primary antidiabetic action through suppression of gluconeogenesis in the liver. The mechanism is based on inhibiting the function of mitochondrial respiratory complex I in hepatocytes and it leads to the activation of AMPK (AMP-activated protein kinase) [16]. Metformin triggers the activation of AMPK (AMP-activated protein kinase), which causes the decrease in hepatic glucose production, the stimulation of glucose uptake in skeletal muscle and a systemic increase in insulin sensitivity [17]. In addition, AMPK inhibits the fat synthesis and also stimulates fat oxidation in the liver [18]. Another mechanism of action of metformin is the inhibition the conversion of lactate to pyruvate, which results in the suppression of gluconeogenic reactions. Biochemical studies show that the the most important metformin' $\mathrm{s}$ target organ in the circulation is the liver. Hepatic processes help to lower glucose concentration in the peripheral blood. The half-life of orally administered metformin in blood is only 3-4 hours. However, the drug remains in the gastrointestinal tract, so its clinical action in normalizing the glucose level in peripheral blood is longer than its half-life [16]. After oral administration, about $70 \%$ of the dose of metformin is absorbed from the small intestine, while the remainder goes to the colon. Metformin does not undergo significant metabolism and it is excreted in the urine unchanged [18]. Metformin also has beneficial 
effects in the prevention of diabetes. In long-term therapy it reduces the risk of developing diabetes by $18 \%$, induces weight loss, causes favourable changes in waist circumference, improves pancreatic beta cell function, reduces the incidence of metabolic syndrome and has positive effects on the cardiovascular system [19].

\subsection{Beneficial impact of metformin on the cardiovascular system}

Metformin, the oldest and most commonly used drug that normalizes glucose level in the peripheral blood, can also be used in the prevention of cardiovascular diseases in patients with T2D. This medicine improves endothelial function, protects against unfavourable remodelling of the cardiac muscle, it also has cardio protective effects and it protects from oxidative stress or chronic inflammation, as well as the negative effects of AT2 (angiotensin II) [20]. It happens through weight loss, improvement in lipid parameters (decrease in concentration of LDL cholesterol and triglycerides), improvement of fibrinolysis efficiency, effects on reduction inflammation or oxidative stress by reducing formation of free radicals in mitochondria, improvement of antioxidant mechanisms, inhibition of the conversion of monocytes to macrophages and stabilization of the atherosclerotic plaque [21]. The studies report that metformin by activating AMPK (AMP-activated protein kinase) inhibits apoptosis of endothelial cells and in this way reduces endothelial cell damage caused by oxidative stress under hyperglycemic conditions. This prevents the formation of atherosclerotic plaques in the blood vessels. Metformin also exerts an anticoagulant activity by inhibiting plasminogen activator inhibitor 1 (PAI-1), which production is stimulated in the conditions of hyperinsulinemia. Administration of metformin during the first 15 minutes of reperfusion after a myocardial infarction has been shown to reduce the infarct size. By activating AMPK, metformin may increase the synthesis of nitric oxide (NO), which is involved in the maintenance of vascular homeostasis. Many nitric oxide (NO) beneficial effects include inhibition of platelet aggregation and therefore protection against the development of coronary artery disease [22]. Increased nitric oxide (NO) synthesis also causes vasodilation in endothelial cells. That has positive impact on the functioning of the vascular endothelium and protects against the development of atherosclerosis. According to the studies metformin treatment at a dose of $150 \mathrm{mg} / \mathrm{kg}$ for 16 weeks significantly reduce serum high-sensitivity Creactive protein (CRP), which is an indicator of chronic inflammation process in the organism. That suggests that metformin indicates anti-inflammatory effect by inhibiting the secretion of pro-inflammatory cytokines [23]. Metformin reduces the size of myocardial infarction and has a beneficial effect on myocardial remodelling after myocardial infarction. In patients with an acute ST-elevation myocardial infarction, peak plasma concentrations of cardiac biomarkers (such as troponin-T) are lower in patients with T2D treated with metformin than in other patients. In addition metformin improves the secondary endpoints left ventricular ejection fraction (LVEF) and left ventricular end systolic diameter (LVESd). These results indicate a cardio protective effect of metformin [24]. Experimental studies and clinical observations provide an increasing number of arguments confirming the numerous benefits of metformin usage in patients with heart failure. The cardio protective properties of metformin are not related to the antihyperglycemic effect, therefore this drug can be also used in the treatment of patients with heart failure without T2D [25]. 


\section{Summary and conclusions}

Metformin is the oldest and most widely used antidiabetic drug, which, despite many years of presence on the pharmaceutical market, still remains as a source of great potential and hope for medical treatment. Current studies reveal that metformin significantly reduces cardiovascular risk in patients with T2D. It is believed that metformin protects against oxidative stress and inflammation, reduces the formation of atherosclerotic plaques, improves endothelial function, indicates anticoagulant effect, reduces the size of myocardial infarction and also has a vasodilating effect by increasing the synthesis of nitric oxide (NO). As a result of its cardio protective effect, metformin could be widely used in the future in patients with heart failure without the features of T2D. According to the statistics cardiovascular diseases are still remaining the leading cause of death both in Poland and in the world. Because of that further researches on effective tools are necessary and required. In the articles presented above metformin has been described as a promising drug which reduces cardiovascular risk and can be used in patients without T2D. This is based on numerous mechanisms of metformin action. Its comprehensive understanding in the future may lead to the improvement in the treatment of cardiovascular diseases. Taking into account the demographic forecasts and statistical data, the Polish society is aging. Both T2D and cardiovascular diseases are disease entities, which probability of incidence increases with age. It means that the number of patients will increase significantly in the nearest future. Therefore, there is a need to conduct further research on the therapeutic benefits of metformin, which could be used in the future both in the prophylaxis and adjunctive therapy of diseases such as myocardial infarction, atherosclerosis or heart failure.

\section{References}

1. Sekcja Niewydolności Serca Polskiego Towarzystwa Kardiologicznego. Niewydolność serca w Polsce - raport 2016. http://www.niewydolnosc-serca.pl/barometr.pdf. 2. Einarson TR, Acs A, Ludwig C, et al. Prevalence of cardiovascular disease in type 2 diabetes: a systematic literature review of scientific evidence from across the world in 20072017. Cardiovasc Diabetol. 2018; 17(1): 83. doi:10.1186/s12933-018-0728-6.

3. Fox C, Golden S, Anderson C, et al. Update on Prevention of Cardiovascular Disease in Adults With Type 2 Diabetes Mellitus in Light of Recent Evidence: A Scientific Statement From the American Heart Association and the American Diabetes Association. Diabetes Care. 2015; 38(9): 1777-1803.

4. Newman J, MD, Schwartzbard A, Weintraub H, et al. Primary Prevention of Cardiovascular Disease in Diabetes Mellitus, J Am Coll Cardiol. 2017; 70(7): 883-893.

5. Einarson TR, Acs A, Ludwig C, et al. Economic Burden of Cardiovascular Disease in Type 2 Diabetes: A Systematic Review. Value Health. 2018; 21(7): 881-890.

6. Ezzati M, Obermeyer Z, Tzoulaki I, et al. The contributions of risk factor trends and medical care to cardiovascular mortality trends, Nat Rev Cardiol. 2015; 12(9): 508-530.

7. Schernthaner G, Lotan C, Baltadzhieva-Trendafilova E, et al. Unrecognised cardiovascular disease in type 2 diabetes: is it time to act earlier?. Cardiovasc Diabetol. 2018; 17(1): 145 . 
8. Strawbridge RJ, van Zuydam NR. Shared Genetic Contribution of Type 2 Diabetes and Cardiovascular Disease: Implications for Prognosis and Treatment, Current Diabetes Reports 2018; 18(8): 59.

9. Gajos G. Diabetes and cardiovascular disease: from new mechanisms to new therapies, Polish Archives of Internal Medicine. 2018; 128(3): 178-186.

10. Bailey CJ. Metformin: historical overview. Diabetologia. 2017; 60(9): 1566-1576.

11. Sanchez-Rangel E, Inzucchi SE. Metformin: clinical use in type 2 diabetes. Diabetologia. 2017; 60(9): 1586-1593.

12. Diabetes Prevention Program Research Group. Long-term safety, tolerability, and weight loss associated with metformin in the Diabetes Prevention Program Outcomes Study. Diabetes Care. 2012; 35(4): 731-737.

13. Holstein A, Stumvoll M. Contraindications can damage your health--is metformin a case in point?. Diabetologia. 2005; 48(12): 2454-2459.

14. Imam TH. Changes in metformin use in chronic kidney disease. Clin Kidney J. 2017; 10(3): 301-304.

15. Inzucchi, SE, Lipska, KJ, Mayo H, et al. Metformin in patients with type 2 diabetes and kidney disease: a systematic review. JAMA. 2014; 312(24): 2668-2675.

16. Minamii T, Nogami M, Ogawa W. Mechanisms of metformin action: In and out of the gut. J Diabetes Investig. 2018; 9(4): 701-703.

17. Spiering MJ. The mystery of metformin. J Biol Chem. 2019; 294(17): 6689-6691.

18. Rena G, Hardie DG, Pearson ER. The mechanisms of action of metformin. Diabetologia. 2017; 60(9): 1577-1585.

19. Aroda VR, Knowler WC, Crandall JP, et al. Metformin for diabetes prevention: insights gained from the Diabetes Prevention Program/Diabetes Prevention Program Outcomes Study. Diabetologia. 2017; 60(9): 1601-1611.

20. Nesti L, Natali A. Metformin effects on the heart and the cardiovascular system: A review of experimental and clinical data. Nutr Metab Cardiovasc Dis. 2017; 27(8): 657-669.

21. Zilov AV, Abdelaziz SI, AlShammary A, et al. Mechanisms of action of metformin with special reference to cardiovascular protection. Diabetes Metab Res Rev. 2019;35(7):e3173. doi: 10.1002/dmrr.3173.

22. Pryor R, Cabreiro F. Repurposing metformin: an old drug with new tricks in its binding pockets. Biochem J. 2015; 471(3): 307-322.

23. Luo F, Das A, Chen J, et al. Metformin in patients with and without diabetes: a paradigm shift in cardiovascular disease management. Cardiovasc Diabetol. 2019; 18(1): 54.

24. Hesen NA, Riksen NP, Aalders B, et al. A systematic review and meta-analysis of the protective effects of metformin in experimental myocardial infarction. PLoS One. 2018; 13(4): e0195858. doi: 10.1371/journal.pone.0183664.

25. Dziubak A, Wójcicka G, Wojtak A, et al. Metabolic Effects of Metformin in the Failing Heart. Int J Mol Sci. 2018; 19(10): 2869. doi: 10.3390/ijms19102869. 